Case Report

\title{
Streptococcus constellatus Causing Septic Thrombophlebitis of the Right Ovarian Vein with Extension into the Inferior Vena Cava
}

\author{
Abdallah Haidar, ${ }^{1}$ Amy Haddad, ${ }^{1}$ Amir Naqvi, ${ }^{1}$ Ngozi U. Onyesoh, ${ }^{1}$ \\ Rushdah Malik, ${ }^{2}$ and Michael Williams ${ }^{1}$ \\ ${ }^{1}$ Department of Internal Medicine, Providence Hospital and Medical Center, 16001 W. Nile Mile Road, Southfield, MI 48075, USA \\ ${ }^{2}$ Department of Infectious Disease, Providence Hospital and Medical Center, 16001 W. Nile Mile Road, Southfield, MI 48075, USA
}

Correspondence should be addressed to Abdallah Haidar; abdhaidar@gmail.com

Received 2 April 2015; Revised 30 May 2015; Accepted 2 June 2015

Academic Editor: Pere Domingo

Copyright (C) 2015 Abdallah Haidar et al. This is an open access article distributed under the Creative Commons Attribution License, which permits unrestricted use, distribution, and reproduction in any medium, provided the original work is properly cited.

\begin{abstract}
Introduction. Streptococcus constellatus collectively with Streptococcus anginosus and Streptococcus intermedius constitute the Streptococcus anginosus (formerly Streptococcus milleri) group. Though they are commonly associated with abscesses, bacteremia with subsequent septic thrombophlebitis is extremely rare, and resulting mortality is infrequent. Case Presentation. We report a case of a previously healthy 60-year-old African American female who presented with Streptococcus constellatus bacteremia associated with septic thrombophlebitis to the right ovarian vein extending into the inferior vena cava. She was urgently treated with antibiotics and anticoagulation. Conclusion. Septic thrombophlebitis has a clinical presentation that is often misleading. Therefore, a high clinical index of suspicion and the use of appropriate imaging modalities (computed tomography) are essential in recognizing and confirming this diagnosis. Prompt treatment is warranted. Surgical thrombectomies have been successfully replaced by a combination of antibiotics and anticoagulation therapy.
\end{abstract}

\section{Case Report}

A 60-year-old African American female with no known prior medical history presented to the emergency department with complaint of intermittent fever, nausea, and vomiting for 2-week duration, in addition to a dry cough for five days. Also notable in her history was a recent travel to Dallas and Las Vegas from Michigan 1 week prior to presentation. Initially, she had a septic presentation with a blood pressure of $82 / 45 \mathrm{~mm} \mathrm{Hg}$, fever of 101 degrees Fahrenheit, leukopenia with a white blood cell count (WBC) of $3.5 \mathrm{k} / \mathrm{mcl}$, a respiratory rate of 22 breaths per minute, and a rapid heart rate of $150 \mathrm{bpm}$. After hemodynamic stabilization with intravenous fluids, a computed tomography (CT) of abdomen and pelvis with contrast was performed and it demonstrated a septic deep venous thrombosis of the ovarian vein with extension into the inferior vena cava (IVC), seen in Figure 1(a). Anticoagulation with intravenous heparin and broad-spectrum antibiotics (ceftriaxone, gentamicin, and metronidazole) were initiated. Blood cultures from two bottles collected prior to antibiotic administration grew Streptococcus constellatus, and the initial antibiotics were changed to ampicillin-sulbactam. The pelvic and transvaginal ultrasound tests were unremarkable. Urine and cervical cultures were negative. Chest X-ray findings included right upper lobe infiltrates concerning pneumonia. CT-thorax demonstrated a 1.1centimeter nodule in the right lung and airspace disease in the right middle lobe suspicious for pneumonia superimposed on emphysema, but it was negative for acute pulmonary embolism. Echocardiogram was normal with no vegetation visualized. The patient remained free of fever for more than 24 hours prior to discharge. She was also bridged after 24 hours to warfarin for continued anticoagulation therapy and remained hemodynamically stable and asymptomatic. She 


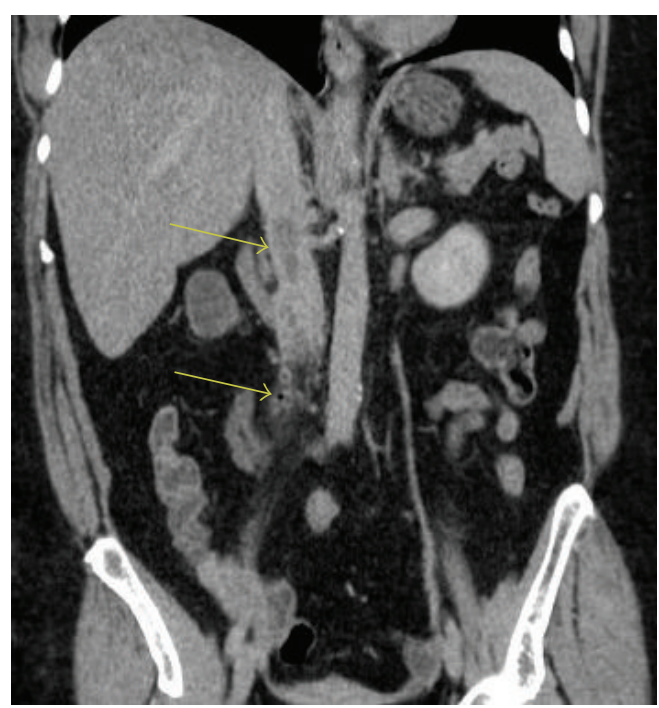

(a)

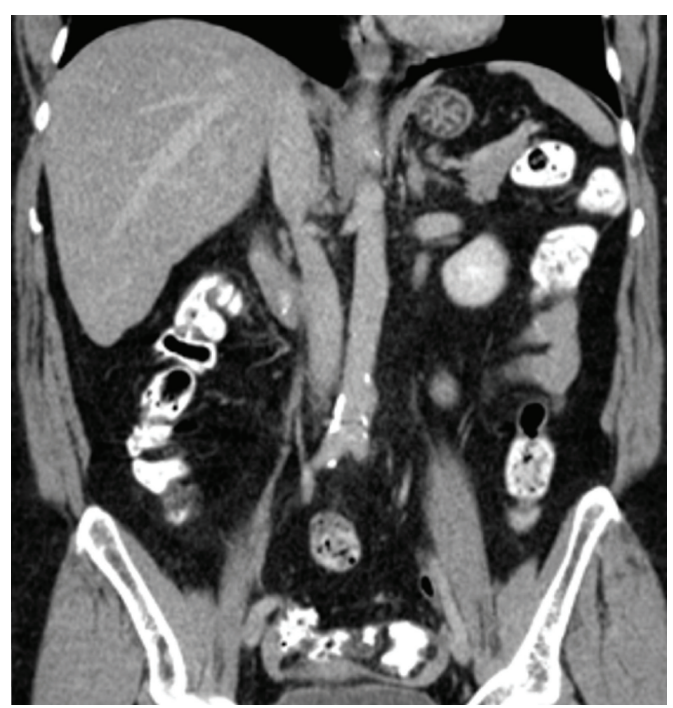

(b)

Figure 1: (a) Septic deep vein thrombosis of the right ovarian vein with extension into the inferior vena cava. Dilation of the right ovarian vein, with a filling defect and air bubble, is seen in the proximal right ovarian vein, surrounded by fat stranding (lower arrow). This filling defect extends into the IVC at the level of the renal veins (upper arrow). These findings are consistent with septic deep thrombophlebitis of the proximal right ovarian vein with extension into the IVC. (b) 2.5 months later, there has been a complete resolution of septic thrombophlebitis of the right ovarian vein.

was discharged home in stable condition after a 5-day hospitalization; she was continued on oral antibiotics (amoxicillin and clindamycin) for 10 days, as well as oral anticoagulation (warfarin) for 3 months. The patient was followed up as an outpatient and was clinically doing well with no complaints and had remained persistently afebrile; her international normalized ratio (INR) remained therapeutic. A repeated CT two and a half months later showed a complete resolution of the septic thrombophlebitis of the right ovarian vein and IVC, Figure 1(b). Also there has been a complete resolution of the pulmonary nodule which may suggest septic emboli to the lung versus upper respiratory infection at presentation.

\section{Background}

Streptococcus constellatus is a species of group C nonhemolytic viridans streptococci. It is gram-positive, nonspore forming, nonmotile, catalase-negative cocci. Streptococcus anginosus, Streptococcus intermedius, and Streptococcus constellatus together make up the anginosus group (formerly milleri group). This group was first found in dental abscesses in 1956 [1]. It is usually considered part of the normal flora of the respiratory, gastrointestinal, and genitourinary tract. However, it can cause systemic purulent infections. Septic thrombophlebitis can be one of its rare clinical presentations, as was the case with our patient. Due to their nonspecific symptoms, including fever and lack of physical findings, early diagnosis of septic thrombophlebitis requires high clinical suspicion as well as the use of appropriate imaging techniques [2]. We present a rare case of septic pelvic thrombophlebitis caused by Streptococcus constellatus in an immunocompetent 60 -year-old female.

\section{Discussion}

Septic pelvic thrombophlebitis is an uncommon entity, with approximately 4 cases occurring per year as seen in one study [13]. Furthermore, cases of septic pelvic thrombophlebitis caused by $S$. constellatus are extremely rare. When it is seen, S. constellatus is more common among intravenous drug abusers $[5,14]$. Up to our knowledge, including our case, there have been eleven cases of septic thrombophlebitis reported in the literature where $S$. constellatus was involved either alone or among other bacteria; see Table 1. The majority of cases were located in the head and neck (7 out of 11); of those, cavernous sinus thrombophlebitis was the most commonly reported location. The remaining four cases were located in the intra-abdominal and pelvic area. Besides this case, three other cases were described: septic iliofemoral thrombophlebitis in an IV drug abuser [5], septic thrombosis of the portal vein and its branches (pylephlebitis) as a complication of diverticulitis [3], and a case of appendicitis that was complicated by portal pylephlebitis [4]. Predisposing factors for those eleven cases were variable.

Other bacteria, such as Staphylococcus aureus, are more commonly associated with septic thrombophlebitis. In a small study, Staphylococcus aureus was found in 4 out of 7 patients who had septic thrombophlebitis, while other bacteria isolated included coagulase-negative Staphylococcus species, Proteus mirabilis, and Propionibacterium species [14]. Streptococcus constellatus has been associated with other infectious presentations including septic shock after tooth extraction [15], mitral and aortic valve endocarditis [16], and subdural empyema [17]. 
TABLE 1: Cases reported in the literature of septic thrombophlebitis with confirmed Streptococcus constellatus involvement.

\begin{tabular}{|c|c|c|c|c|c|}
\hline $\begin{array}{l}\text { Case } \\
\text { number }\end{array}$ & Age & $\operatorname{Sex}(M / F)$ & $\begin{array}{l}\text { Anatomic location/clinical } \\
\text { diagnosis }\end{array}$ & Predisposing factors & Source \\
\hline 1 & 60 & $\mathrm{~F}$ & $\begin{array}{l}\text { Ovarian vein with an extension into } \\
\text { inferior vena cava }\end{array}$ & Possibly travel and dehydration & This case report \\
\hline 2 & 63 & M & Portal vein thrombosis & $\begin{array}{l}\text { Complication of perforated } \\
\text { diverticulitis }\end{array}$ & Van De Wauwer and Irvin, 2005 [3] \\
\hline 3 & 13 & M & Portal vein thrombosis & Complication of appendicitis & Sakalkale and Reeve, 2006 [4] \\
\hline 4 & 24 & M & Iliofemoral deep vein thrombosis & Intravenous drug abuse & Sulaiman et al., 2011 [5] \\
\hline 5 & 56 & $\mathrm{~F}$ & $\begin{array}{l}\text { Cavernous sinus thrombosis } \\
\text { complicated by narrowing of the } \\
\text { internal carotid artery, } \\
\text { subarachnoid abscess, and multiple } \\
\text { pulmonary septic emboli }\end{array}$ & No contributory medical history & Hoshino et al., 2007 [6] \\
\hline 6 & 39 & M & Cavernous sinus thrombosis & Chronic alcohol consumption & Chang et al., 2003 [7] \\
\hline 7 & 54 & $\mathrm{~F}$ & $\begin{array}{l}\text { Facial abscess, cavernous sinus } \\
\text { thrombosis (CST), bilateral internal } \\
\text { jugular thrombosis, and multiple } \\
\text { lung abscesses }\end{array}$ & $\begin{array}{l}\text { Mandibular dental infection, } \\
\text { immunosuppressed }\end{array}$ & Jones and Arnold, 2009 [8] \\
\hline 8 & 45 & M & Cavernous sinus thrombosis & Severe periodontitis & Imholz et al., 2014 [9] \\
\hline 9 & 51 & $\mathrm{~F}$ & $\begin{array}{l}\text { Cavernous sinus, maxillary vein, } \\
\text { and multiple pulmonary nodular } \\
\text { lesions (a Lemierre syndrome } \\
\text { variant) }\end{array}$ & Acute otolaryngologic infection & Yamaguchi et al., 2010 [10] \\
\hline 10 & 56 & M & Cavernous sinus thrombosis & $\begin{array}{l}\text { Unknown origin, possibly prior } \\
\text { endoscopic retrograde biliary } \\
\text { drainage }\end{array}$ & Hung et al., 2014 [11] \\
\hline 11 & 52 & $\mathrm{~F}$ & $\begin{array}{l}\text { Cavernous sinus thrombosis and } \\
\text { meningitis }\end{array}$ & $\begin{array}{l}\text { Chronic sinusitis and } \\
\text { complication of osteoporosis, } \\
\text { including orthopedic surgery }\end{array}$ & Chung et al., 2014 [12] \\
\hline
\end{tabular}

S. constellatus had previously been reported more frequently as part of the milleri group (currently anginosus group). In a UK study [18], 151 strains of milleri group were collected from multiple laboratories. There have been 33 total cases of $S$. constellatus previously identified, and no predominant body site was found to be associated with this organism. Three cases were associated with genitourinary clinical source and four were isolated from other abdominal and pelvic sites. On the other hand, the total number of S. anginosus identified was 89 , with predominant source being the genitourinary and gastrointestinal tracts, 40 and 19 , respectively. The infections of the central nervous system (CNS) were most commonly associated with $S$. intermedius strains; 18 out of 29 total strains were identified.

Streptococcus constellatus has often been associated with abscesses. Clarridge III et al. [19] reported 118 patients with confirmed isolates of the Streptococcus milleri group, of which 54 patients (46\%) had a total of 56 isolates of S. constellatus. In $41(73 \%)$ of the 56 isolates obtained, S. constellatus was involved in abscess formation.

Bacteremia from the Streptococcus anginosus group is infrequent. Knowledge of the different clinical presentations may aid in determining the source of the infection. In our case, however, the exact etiology and source of septic thrombophlebitis of the right ovarian vein remained unclear. Dehydration and recent travel history could have been the predisposing factors contributing to deep venous thrombosis (DVT) in our case. In general, septic pelvic thrombophlebitis is seen more often among younger females in their reproductive age. Its risk factors include underlying malignancy, pelvic infection, uterine fibroids, and severe dehydration. Other factors include cesarean section, pregnancy, hormonal stimulation, induced abortion, and a postpartum state in nonmenopausal females, none of which was present in our patient. Ovarian vein thrombosis and septic pelvic thrombophlebitis have predominantly been described in young women who are postpartum or after surgery compared to our case in a postmenopausal woman. The clinical presentation related to these entities has been reported to include ipsilateral abdominal pain, fever, flank or back pain, nausea or vomiting, and leukocytosis on laboratory findings [20-22].

Patients with septic thrombophlebitis are at risk of pulmonary embolism, especially when there is involvement of the IVC. Therefore, immediate treatment is important. Previously, the treatment of septic thrombophlebitis involved surgery [23]. However, anticoagulation and antibiotic therapies have since been proven to be efficacious in the treatment of septic thrombophlebitis, with no recurrence after treatment [14]. Broad-spectrum antibiotics are recommended as part of the initial treatment course. Blood cultures are frequently negative, making it difficult to define commonly responsible organisms. The most commonly involved 
pathogens are E. coli, Bacteroides spp., other coliforms, or streptococci. S. aureus is less common without the appropriate predisposing risk factors. Gram-negative pathogens are seen more often in patients with intra-abdominal pathology [24]. $14 \%$ of the cases have had multiple causative agents isolated. One case series involved 46 patients with septic pelvic thrombophlebitis, including seven patients with significant involvement of the ovarian vein. In this report, the treatment course associated chloramphenicol and penicillin for antimicrobial coverage and heparin for anticoagulation. Of the 46 patients described, 42 patients were subsequently without fever within 7 days (mean: 2.5 days) [25]. The duration of intravenous therapy can be as short as 7 days if there are no other complications, followed by a course of oral antibiotics. There is a lack of definitive studies or recommendations to guide the duration of anticoagulation therapy for this entity, but one source recommends the use of enoxaparin at a dose of $1 \mathrm{mg} / \mathrm{kg}$ daily or warfarin with a goal INR of 2.5 for 3-6-month duration if the thrombosis involves the ovarian vein. This can be followed by a repeat CT scan to determine resolution of the clot after 3 months, at which point anticoagulation can be discontinued if CT is negative or continued for an additional 3 months if CT is positive [2]. Surgical thrombectomies are typically reserved for cases that fail conservative management.

\section{Conclusion}

Septic pelvic thrombophlebitis, although rare, can be associated with high morbidity. Knowing the underlying etiologies and possible masquerading presentation will help in its recognition. Instituting diagnostic techniques such as CT will also aid in its diagnosis. Rapid institution of therapy including the appropriate antibiotic in combination with anticoagulation is recommended. Further studies are needed on the duration of anticoagulation therapy. Surgery is no longer first line of therapy. Underlying etiologies should be investigated. Overall, prognosis is favorable if detected and treated early.

\section{Conflict of Interests}

The authors declare that there is no conflict of interests regarding the publication of this paper.

\section{References}

[1] O. Guthof, "Pathogenic strains of Streptococcus viridans; streptocci found in dental abscesses and infiltrates in the region of the oral cavity," Zentralblatt für Bakteriologie, Parasitenkunde, Infektionskrankheiten und Hygiene. 1. Abt. Medizinisch-hygienische Bakteriologie, Virusforschung und Parasitologie. Originale, vol. 166, no. 7-8, pp. 553-564, 1956.

[2] J. Garcia, R. Aboujaoude, J. Apuzzio, and J. R. Alvarez, "Septic pelvic thrombophlebitis: diagnosis and management," Infectious Diseases in Obstetrics and Gynecology, vol. 2006, Article ID 15614, 4 pages, 2006.

[3] C. Van De Wauwer and T. T. Irvin, "Pylephlebitis due to perforated diverticulitis," Acta Chirurgica Belgica, vol. 105, no. 2, pp. 229-230, 2005.
[4] R. P. Sakalkale and P. Reeve, "Portal venous thrombophlebitis in a case of perforated appendicitis: lessons from a case," New Zealand Medical Journal, vol. 119, no. 1234, Article ID U1984, 2006.

[5] L. Sulaiman, J. Hunter, F. Farquharson, and H. Reddy, "Mechanical thrombectomy of an infected deep venous thrombosis: a novel technique of source control in sepsis," British journal of anaesthesia, vol. 106, no. 1, pp. 65-68, 2011.

[6] C. Hoshino, N. Satoh, S. Sugawara, C. Kuriyama, A. Kikuchi, and M. Ohta, "Septic cavernous sinus thrombosis complicated by narrowing of the internal carotid artery, subarachnoid abscess and multiple pulmonary septic emboli," Internal Medicine, vol. 46, no. 6, pp. 317-323, 2007.

[7] W.-N. Chang, S.-D. Chen, C.-C. Lui, C.-R. Huang, and C.-H. Lu, "Septic cavernous sinus thrombosis due to streptococcus constellatus infection," Journal of the Formosan Medical Association, vol. 102, no. 10, pp. 733-736, 2003.

[8] R. G. Jones and B. Arnold, "Suddenonset proptosis secondary to cavernous sinus thrombosis from underlying mandibular dental infection," BMJ Case Reports, 2009.

[9] B. Imholz, M. Becker, T. Lombardi, and P. Scolozzi, "Septic thrombosis of the cavernous sinus secondary to a Streptococcus milleri oral infection," Dentomaxillofacial Radiology, vol. 41, no. 6, 2014.

[10] M. Yamaguchi, H. Nishizawa, T. Yasumoto et al., "Lemierre syndrome with blepharoptosis," Internal Medicine, vol. 49, no. 8, pp. 753-757, 2010.

[11] J.-H. Hung, C.-Y. Chien, W.-Y. Chen, Y.-H. Shih, and Y.-S. Chang, "Cavernous sinus thrombosis caused by an unknown hematogenous origin: a case report," 2014, http://www.digitalred.com.tw/2014oph4/web_pdf/PO-200.pdf.

[12] H. C. Chung, S. H. Park, E. S. Kim, Y. I. Kim, S. H. Lee, and I. S. Nam-Goong, "A case of cavernous sinus thrombophlebitis and meningitis as a complication in osteopetrosis," Journal of Bone Metabolism, vol. 21, no. 3, pp. 227-232, 2014.

[13] A. G. Witlin, B. M. Mercer, and B. M. Sibai, "Septic pelvic thrombophlebitis or refractory postpartum fever of undetermined etiology," Journal of Maternal-Fetal and Neonatal Medicine, vol. 5, no. 6, pp. 355-358, 1996.

[14] A. K. Ang and O. W. Brown, "Septic deep vein thrombosis," Journal of Vascular Surgery, vol. 4, no. 6, pp. 563-566, 1986.

[15] K. W. P. Ng and A. Mukhopadhyay, "Streptococcus constellatus bacteremia causing septic shock following tooth extraction: a case report," Cases Journal, vol. 2, article 6493, 2009.

[16] G. Concistré, F. Chiaramonti, A. Miceli, and M. Glauber, "Mitral and aortic valve endocarditis caused by a rare pathogen: Streptococcus constellatus," Interactive Cardiovascular and Thoracic Surgery, vol. 14, no. 6, pp. 889-890, 2012.

[17] A. Bouziri, A. Khaldi, H. Smaoui, K. Menif, and N. B. Jaballah, "Fatal subdural empyema caused by Streptococcus constellatus and Actinomyces viscosus in a child-case report," Journal of Microbiology, Immunology and Infection, vol. 44, no. 5, pp. 394396, 2011.

[18] R. A. Whiley, D. Beighton, T. G. Winstanley, H. Y. Fraser, and J. M. Hardie, "Streptococcus intermedius, Streptococcus constellatus, and Streptococcus anginosus (the Streptococcus milleri group): association with different body sites and clinical infections," Journal of Clinical Microbiology, vol. 30, no. 1, pp. 243-244, 1992.

[19] J. E. Clarridge III, S. Attorri, D. M. Musher, J. Hebert, and S. Dunbar, "Streptococcus intermedius, Streptococcus constellatus, 
and Streptococcus anginosus ('Streptococcus milleri group') are of different clinical importance and are not equally associated with abscess," Clinical Infectious Diseases, vol. 32, no. 10, pp. 1511-1515, 2001.

[20] G. Ventolini and E. L. Mathews, "Septic pelvic thrombophlebitis: an update," Female Patient, vol. 31, p. 35, 2006.

[21] A. G. Adesiyun, M. O. A. Samaila, and A. Ojabo, "Postpartum ovarian vein thrombosis: incidental diagnosis at surgery," Case Reports in Obstetrics and Gynecology, vol. 2014, Article ID 898342, 3 pages, 2014.

[22] R. B. Chebl, S. Krupp, K. Bourgi, and G. A. Dagher, "Idiopathic ovarian vein thrombosis in the postmenopausal age," International Journal of Case Reports and Images, vol. 4, no. 11, pp. 611614, 2013.

[23] R. Hassen-Khodja, J.-Y. Gillet, M. Batt et al., “Thrombophlebitis of the ovarian vein with free-floating thrombus in the inferior vena cava," Annals of Vascular Surgery, vol. 7, no. 6, pp. 582-586, 1993.

[24] R. A. Johnson, R. A. Zajac, and M. E. Evans, "Suppurative thrombophlebitis: correlation between pathogen and underlying disease," Infection Control, vol. 7, no. 12, pp. 582-585, 1986.

[25] W. E. Josey, "Heparin therapy in septic pelvic thrombophlebitis: a study of 46 cases," The American Journal of Obstetrics and Gynecology, vol. 120, no. 2, pp. 228-233, 1974. 


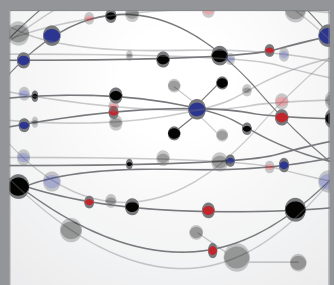

The Scientific World Journal
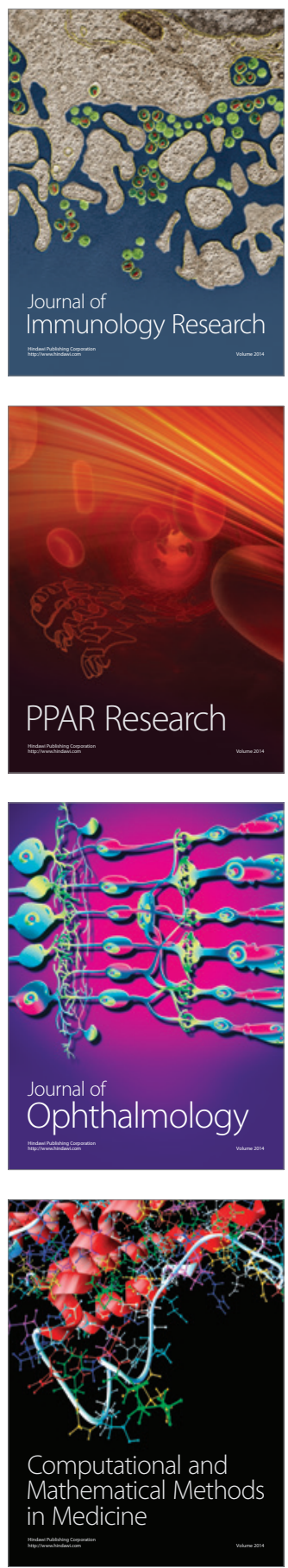

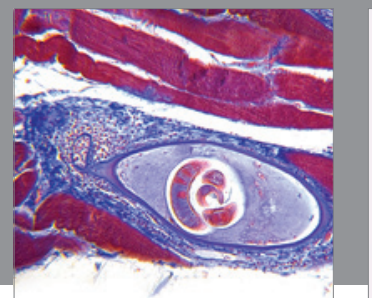

Gastroenterology

Research and Practice
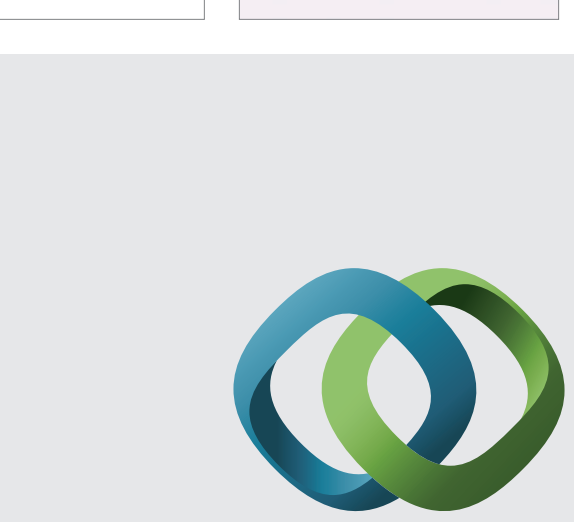

\section{Hindawi}

Submit your manuscripts at

http://www.hindawi.com
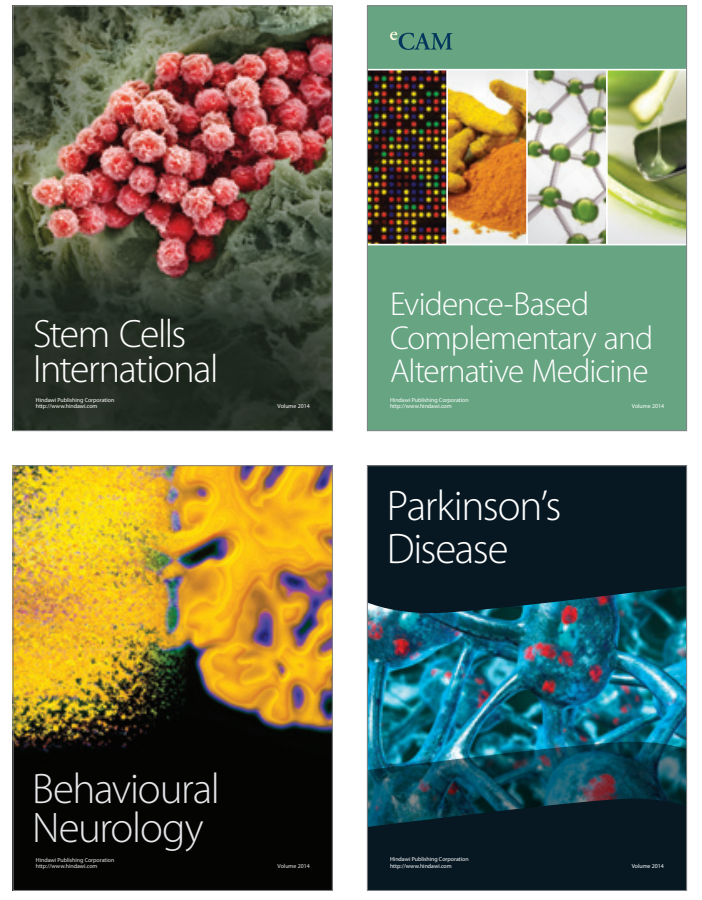
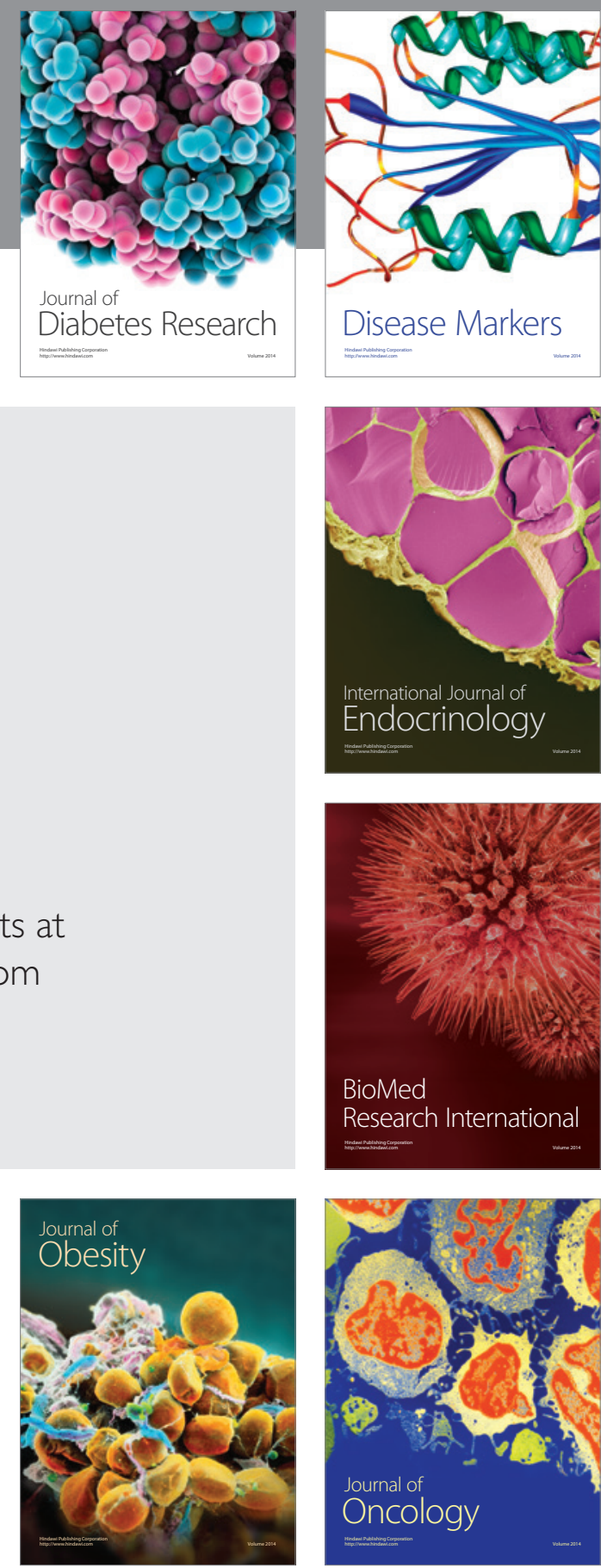

Disease Markers
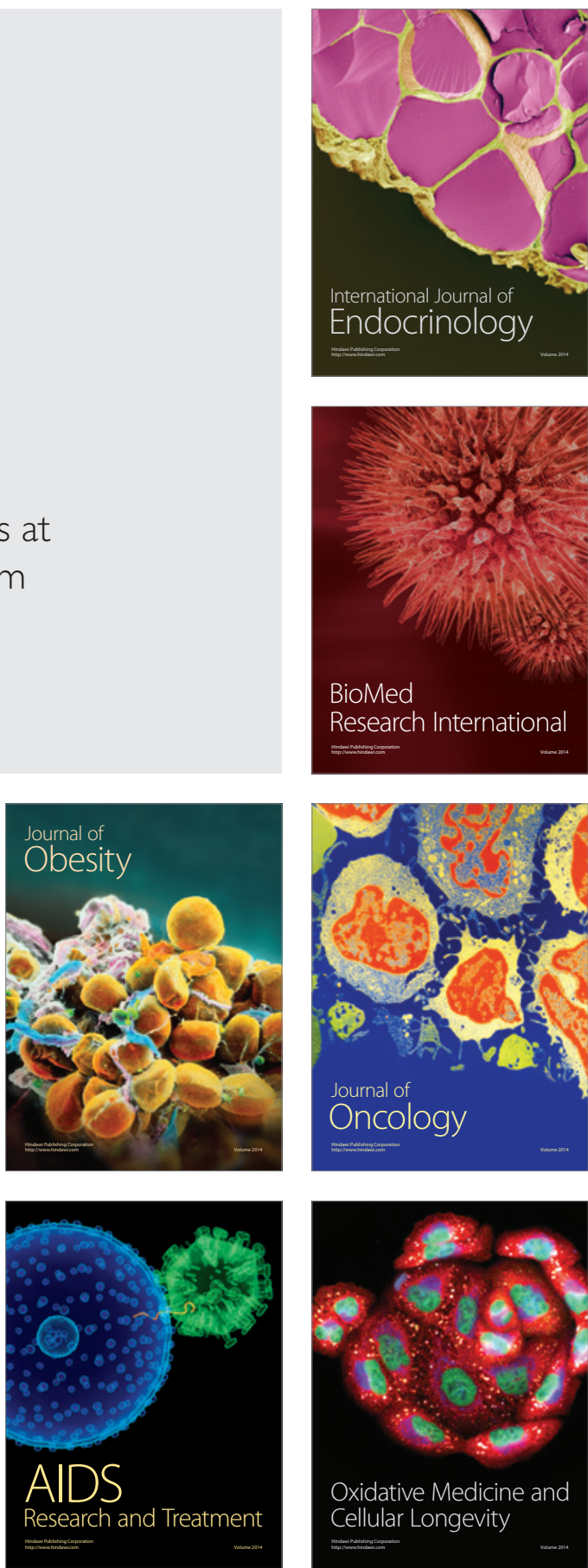